\title{
A simple passive device for drag reduction over a three dimensional Ahmed body model
}

\author{
Naseeb Ahmed Siddiqui ${ }^{1}$, Martin Agelin-Chaab ${ }^{2}$ \\ ${ }^{1}$ Department of Automotive, Mechanical and Manufacturing Engineering, Ontario Tech University, Oshawa, Canada \\ ${ }^{21}$ Department of Automotive, Mechanical and Manufacturing Engineering, Ontario Tech University, Oshawa, Canada \\ *naseeb.siddiqui@ontariotechu.net
}

\begin{abstract}
Experimental investigation on a generic vehicle model developed by (Ahmed et al., 1984) reports the salient features of the overall aerodynamics around the vehicle. The importance and contribution of the pressure drag of the vehicle's back end have been explored by altering the angle of the slanted surface. The breakdown of the drag on the body revealed that almost $85 \%$ of aerodynamic drag is the pressure drag, and the rear end contributes to the significant part of it. Therefore, several works were dedicated to control or manipulate the flow at the slanted surface to reduce the pressure drag. There are two methods to influence the flow features at the slant surface. One is to suppress the bubbles developed at the slanted surface, and the second is to attenuate the strength of the induced drag due to side edges. Encouraging drag reductions have been recorded for both methods. The current article introduces a simple passive device in the form of a rectangular flap. The device is tested over the $35^{\circ}$ Ahmed model and is installed at the slanted surface. The angle formed between the slant surface of the base model and the rectangular flap is varied from $0^{\circ}$ to $25^{\circ}$ to find out the optimum angle for the maximum drag reduction. The salient features of this passive device have been investigated using the Reynolds-Averaged Naiver-stokes equation with an SST k-omega turbulence closure model on a commercial Fluent solver. The Reynolds number is $7.8 \times 10^{5}$, based on the height of the model. The numerical simulation revealed that the increase in flap angle from $0^{\circ}$ to $10^{\circ}$ provides a significant amount of drag reduction, which reached a maximum of $14.3 \%$ for a $10^{\circ}$ flap model. However, further, an increase in flap angle after $10^{\circ}$ reports a consistent drop in the drag reduction. This result is exciting because, based on the existing studies using small flaps, it was guessed that the use of flap would discourage the induced drag and would be the main reason for drag reduction. However, it was found that the reason for significant drag reduction is the suppression of the bubbles developed at the slant surface and the bubbles just near the rear end. The objective of this article is to reveal the reasons behind this counter-intuitive drag reduction due to a simple flap device.
\end{abstract}

Word count: 377 